\title{
An imperfect quality items with learning and inflation under two limited storage capacity
}

\author{
S.R. Singh ${ }^{a}$, Shalini Jain ${ }^{b^{*}}$ and S. Pareek ${ }^{b}$
}

${ }^{a}$ Department of Mathematics, D.N. College, Meerut, India

${ }^{b}$ Centre for Mathematical Sciences, Banasthali University, Rajasthan, India

\section{H R O N I C L E}

\begin{tabular}{l}
\hline Article history: \\
Received February 22013 \\
Received in revised format \\
May $28 \quad 2013$ \\
Accepted June 222013 \\
Available online \\
June 262013 \\
\hline Keywords: \\
EPQ \\
Two- warehouse \\
Imperfect \\
Shortages \\
Weibull deterioration \\
Learning \\
Salvage value
\end{tabular}

\section{A B S T R A C T}

In this paper, we develop a two-warehouse imperfect production model under two cases: (i) model starts with shortages (ii) model ends with shortages. Most of the researchers proposed the models for perfect items but we develop for imperfect quality items, which is very realistic. Demand is taken as time dependent and dependent on the production. Holding cost in rented warehouse $(\mathrm{RW})$ is greater than own warehouse $(\mathrm{OW})$. Deterioration is taken as Weibull distribution in both OW and RW. Shortages are allowed and partially backlogged. The effect of learning on production cost is also considered. Learning from one cycle to other cycle, improve the efficiency of the organization. A numerical example including the sensitivity analysis is given to validate the results of the production-inventory model.

\section{Introduction}

The classical inventory models usually assume the available warehouse has unlimited capacity. In many practical situations, there exist many factors like temporary price discounts making retailers buy a capacity of goods exceeding their own warehouse (OW). In this case, retailers will either rent other warehouses or rebuild a new warehouse. However, from economical point of views, they usually choose to rent other warehouses. Hence, an additional storages space known as rented warehouses (RW) is often required due to limited capacity of showroom facility. In recent years, various researchers have discussed a two-warehouse inventory system. Therefore, due to the limited capacity of the available showroom facility (existing storage, own warehouse $(\mathrm{OW})$ ), an additional storage which is assumed to be available with abundant space is required to hold a large stock. This additional storage facility may be a rented warehouse (RW) with better preserving facility. This is first proposed by Hartely (1976). In this system, it is assumed that the holding cost in RW is greater than that in OW.

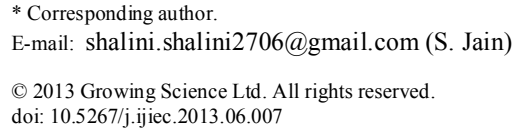


Hence, items in RW are first transferred to OW to meet the demand until the stock level in RW drops to zero and then items in OW are released.

By assuming constant demand rate, Sarma (1987) developed a deterministic inventory model for a single deteriorating item with shortages and two levels of storage. Pakkala and Achary (1992) extended the two-warehouse inventory model for deteriorating items with finite replenishment rate and shortages. Besides, the ideas of time-varying demand for deteriorating items with two storage facilities were considered by Benkherouf (1997) and Bhunia and Maiti (1998). Singh et al. (2008) provided a two-ware inventory model for deteriorating items. In that model shortages are allowed and partially backlogged. Singh et al.(2009) offered a two-warehouse inventory model for deteriorating items with shortages under inflation and time-value of money. Recently, Jaggi and Verma (2010) developed a two-warehouse inventory model with linear trend in demand under the inflationary conditions. Shortage was allowed and completely backlogged.

Most of the existing EOQ models unrealistically ignored the presence of the imperfect production process and equipment. Porteus (1986) and Rosenblatt and Lee (1986) was the first who developed a model with imperfect quality items. Furthermore, various researchers have discussed a two-warehouse inventory system. Kimand Hong (1999) determined the optimal production run length in deteriorating production process. Salameh and jaber (2000) developed an economic production/ inventory quantity model for items with imperfect quality. They assumed that poor-quality items are sold as a single batch by the end of the $100 \%$ screening process. Goyal et al. (2002) extended the model of Salameh and Jaber (2000) to develop a practical approach to determine the EPQ for items with imperfect quality. Chung and Hou (2003) developed a model to determine an optimal run time for a deteriorating production system with shortages.

Papachristos and Konstantaras (2006) developed economic ordering quantity models for items with imperfect quality and discussed many of the assumption of Salameh and Jaber (2000). Huang (2004) and Chung and Huang (2006) investigated the model of Salameh and Jaber (2000) in a two-level supply chain (vendor-buyer), while Wee et al. (2007) and Eroglu and Ozdemir (2007) independently extended it by allowing for shortages. In addition, Chan et al. (2003) develop an economic production model using similar assumptions as Salameh and Jaber (2000). Jaber et al. (2008) develop the model by using the assumption of Salameh and Jaber (2000) and discussed the effect of learning effects. In the classical economic production/order quantity models, the items produced/ received are implicitly assumed to be with perfect quality. However, it may not always be the case. Due to imperfect production process, natural disasters, damage or breakage in transit, or for many other reasons, the lot sizes produced/ received may contain some defective items.

Goyal and Giri (2003) considered the production-inventory problem with time varying demand, production and deterioration rate. Salameh and Jaber (2000) developed an economic production/ inventory quantity model for items with imperfect quality. Goyal et al. (2002) extended the model of Salameh and Jaber (2000) to develop a practical approach to determine the EPQ for items with imperfect quality. Chun et al. (2009) developed a two warehouse model with imperfect quality. Recently Singh et al. (2012) proposed a warehouse imperfect fuzzified production model with shortages and inflation.

There are lots of real life problem where the defective rate, ordering cost are decreases from one cycle to other. Such as automotive manufacturing for shipments of raw material where the percentage of defective items per lot decreases with cumulative number of shipments conforming to a learning curve and the demand of raw material is highly uncertain due to inflation and market complexities. We developed the models where percentage of defective items in each lot, production cost are follows learning effects. Most of the papers are develop for perfect quality items. But In this paper, we developed a two warehouse model with imperfect quality items with learning effect which is more realistic. We consider the two models (i) shortages at the end and, (ii) starts with the shortages. We 
assume that demand is time- dependent and deterioration is taken as Weibull for both OW and RW. Shortages are also allowed in this model.

\section{Assumptions and Notations}

\subsection{Assumptions}

In developing the mathematical models of the inventory system the following assumptions are used:

1. The demand rate $D(t)$ is deterministic and is a known function of time; the function $D(t)$ is given by:

$D(t)=a e^{b t}$, where $a$ and $b>0$.

2. Production rate is dependent on the demand rate i.e. $P=k d=k a e^{b t}$

3. Shortages are allowed and partially backlogged where $B=e^{-\delta t}, \delta$ is a backlogging parameter, $\delta>0$.

4. Salvage value is associated to deteriorated units during the cycle time.

5. The time horizon of the inventory system is infinite.

6. Replenishment rate is infinite, and lead-time is zero.

7. The owned warehouse (OW) has a fixed capacity of $\mathrm{W}$ units, the rented warehouse (RW) has unlimited capacity.

8. The goods of OW are consumed only after consuming the goods kept in RW.

9. The unit inventory costs (including holding cost and deterioration cost) per unit time in RW are higher than those in OW.

10. The deterioration rate is taken as weibull in both OW and RW.

In addition, the following notations are used throughout this study:

\subsection{Notations}

W Fixed capacity level of $\mathrm{OW}$

$\alpha \quad$ Scale parameter of the deterioration rate in $\mathrm{OW}$

$\beta \quad$ Shape parameter of the deterioration rate in OW

$a, b \quad$ Parameters of the demand rate

$\left(C_{p}+\frac{C_{0}}{n^{\delta}}\right) \quad$ Production cost with learning effect

$C_{R W} \quad$ Present worth of Holding cost in RW

$C_{O W}$ Present worth of Holding cost in OW

$C_{3} \quad$ Present worth of Deterioration cost

$C_{4} \quad$ Present worth of Opportunity cost

$C_{s} \quad$ Present worth of Shortage cost

$C_{5} \quad$ Present worth of Rework cost 
$f(X) \quad$ Probability density function of $\mathrm{X}$

$I_{i 1} \quad$ Inventory level in OW at time t with $t \in\left[0, t_{1}\right]$

$I_{i 2}$ Inventory level in RW at time $\mathrm{t}$ with $t \in\left[t_{1}, t_{2}\right]$

$I_{i 3} \quad$ Inventory level in RW at time $\mathrm{t}$ with $t \in\left[t_{2}, t_{3}\right]$

$I_{i 4} \quad$ Inventory level in OW at time t with $t \in\left[t_{1}, t_{3}\right]$

$I_{i 5} \quad$ Inventory level in OW at time $\mathrm{t}$ with $t \in\left[t_{3}, t_{4}\right]$

$I_{i 6} \quad$ Inventory level in OW at time $\mathrm{t}$ with $t \in\left[t_{4}, t_{5}\right]$

$I_{i 7} \quad$ Inventory level in OW at time t with $t \in\left[t_{5}, T\right]$

\section{Formulation of the model}

In Fig.1, the inventory level during a production cycle in which both OW and RW are used. Initially, the inventory level is zero. The production starts at time $\mathrm{t}=0$ and items accumulate from 0 up to $\mathrm{W}$ units in $\mathrm{OW}$ in $\mathrm{t}_{1}$ units of time. After time $\mathrm{t}_{1}$ any production quantity exceeding $\mathrm{W}$ will be stored in $\mathrm{RW}$. After this production stopped and the inventory level in RW begins to decrease at $t_{2}$ and will reach 0 units at $t_{3}$ because of demand and deterioration. The inventory level in OW comes to decrease at $t_{1}$ and then falls below $\mathrm{W}$ at $\mathrm{t}_{3}$ due to deterioration. The remaining stocks in $\mathrm{OW}$ will be fully exhausted at $\mathrm{t}_{4}$ owing to demand and deterioration, the inventory becomes zero. At this time shortage starts developing and at time $t_{5}$ it reaches to maximum shortage level, at this time fresh production starts to clear the backlog by the time $\mathrm{T}$.

\subsection{Model I: When shortages at the end}

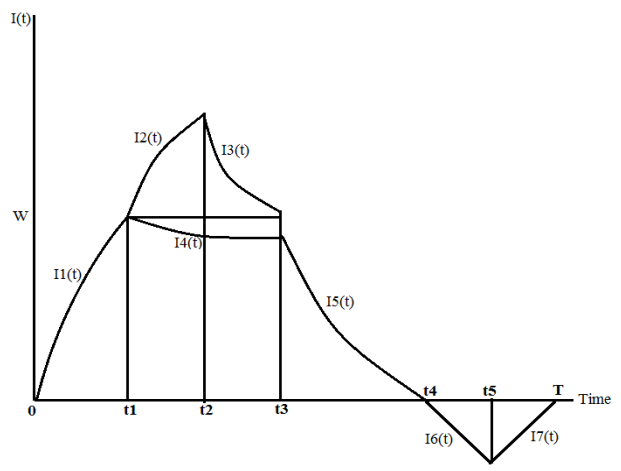

Fig. 1. Two warehouse model with the shortages at the end

$$
\begin{array}{ll}
I_{1}^{\prime}(t)+\alpha \beta t^{\beta-1} I_{1}(t)=P-D & 0 \leq t \leq t_{1} \\
I_{2}^{\prime}(t)+\alpha \beta t^{\beta-1} I_{2}(t)=P-D & t_{1} \leq t \leq t_{2} \\
I_{3}^{\prime}(t)+\alpha \beta t^{\beta-1} I_{3}(t)=-D & t_{2} \leq t \leq t_{3} \\
I_{4}^{\prime}(t)+\alpha \beta t^{\beta-1} I_{5}(t)=0 & t_{1} \leq t \leq t_{3} \\
I_{5}^{\prime}(t)+\alpha \beta t^{\beta-1} I_{4}(t)=-D & t_{3} \leq t \leq t_{4} \\
I_{6}^{\prime}(t)=-B D & t_{4} \leq t \leq t_{5} \\
I_{7}^{\prime}(t)=P-D & t_{5} \leq t \leq T
\end{array}
$$

with these boundary conditions 


$$
\begin{aligned}
& I_{1}(0)=0, I_{2}\left(t_{1}\right)=0, I_{3}\left(t_{3}\right)=0, I_{4}\left(t_{1}\right)=W, I_{5}\left(t_{4}\right)=W, I_{6}\left(t_{4}\right)=0, \text { and } I_{7}(T)=0, \\
& I_{1}(t)=(k-1) a\left[t+\frac{b t^{2}}{2}+\frac{\alpha t^{\beta+1}}{\beta+1}+\frac{\alpha b t^{\beta+2}}{\beta+2}\right]\left(1-\alpha t^{\beta}\right) \\
& I_{2}(t)=(k-1) a\left[\left(t-t_{1}\right)+\frac{b}{2}\left(t^{2}-t_{1}^{2}\right)+\frac{\alpha}{\beta+1}\left(t^{\beta+1}-t_{1}^{\beta+1}\right)+\frac{b \alpha}{\beta+2}\left(t^{\beta+2}-t_{1}^{\beta+2}\right)\right]\left(1-\alpha t^{\beta}\right) \\
& I_{3}(t)=a\left[\left(t_{3}-t\right)+\frac{b}{2}\left(t_{3}{ }^{2}-t^{2}\right)-\frac{\alpha}{\beta+1}\left(t_{3}^{\beta+1}-t^{\beta+1}\right)-\frac{b \alpha}{\beta+2}\left(t_{3}^{\beta+2}-t^{\beta+2}\right)\right]\left(1-\alpha t^{\beta}\right) \\
& I_{4}(t)=W e^{\alpha\left(t_{1}^{\beta}-t^{\beta}\right)} \\
& I_{5}(t)=a\left[\left(t_{4}-t\right)+\frac{b}{2}\left(t_{4}{ }^{2}-t^{2}\right)-\frac{\alpha}{\beta+1}\left(t_{4}^{\beta+1}-t^{\beta+1}\right)+\frac{b \alpha}{\beta+2}\left(t_{4}^{\beta+2}-t^{\beta+2}\right)\right]\left(1-\alpha t^{\beta}\right) \\
& I_{6}(t)=a\left[t_{4}+\frac{(b-\delta) t_{4}^{2}}{2}-\frac{b \delta t_{4}^{3}}{3}\right] \\
& I_{7}(t)=(k-1) a\left[(t-T)+\frac{b}{2}\left(t^{2}-T^{2}\right)\right] \\
& P r O d u c t i o n
\end{aligned}
$$

Production cost

$$
P C=\left(C_{p}+\frac{C_{0}}{n^{\gamma}}\right) \int_{0}^{t_{2}} k a e^{b t} e^{-r t} d t=\left(C_{p}+\frac{C_{0}}{n^{\gamma}}\right) k a\left(t_{2}+\frac{(b-r) t_{2}^{2}}{2}-\frac{b r t_{2}^{2}}{2}\right)
$$

Present worth of Holding cost in $R W$

$$
\begin{aligned}
& H_{R W}=C_{R W}\left\{\int_{t_{1}}^{t_{2}} I_{2}(t) e^{-r t} d t+\int_{t_{2}}^{t_{3}} I_{3}(t) e^{-r t} d t\right\} \\
& =a C_{R W}\left[(k-1)\left\{\left(\frac{t_{2}^{2}}{2}+\frac{t_{1}^{2}}{2}\right)-r\left(\frac{t_{2}^{3}}{3}+\frac{t_{1}^{3}}{6}\right)+\frac{b}{2}\left(\frac{t_{2}^{3}}{3}+\frac{2 t_{1}^{3}}{3}\right)\right\}+\left\{\left(\frac{t_{2}^{2}}{2}+\frac{t_{3}^{2}}{2}\right)-r\left(\frac{t_{2}^{3}}{3}+\frac{t_{3}^{3}}{6}\right)+\frac{b}{2}\left(\frac{t_{2}^{3}}{3}+\frac{2 t_{3}^{3}}{3}\right)\right\}\right]
\end{aligned}
$$

Present worth of Holding cost in $\mathrm{OW}$

$$
\begin{aligned}
& H_{O W}=C_{O W}\left\{\int_{0}^{t_{1}} I_{1}(t) e^{-r t} d t+\int_{t_{1}}^{t_{3}} I_{5}(t) e^{-r t} d t+\int_{t_{3}}^{t_{4}} I_{4}(t) e^{-r t} d t\right\} \\
& =C_{O W}\left[\begin{array}{l}
\left.(k-1) a\left\{\frac{t_{1}^{2}}{2}-\frac{r t_{1}^{3}}{3}+\frac{b t_{1}^{3}}{6}-\frac{b r t_{1}^{4}}{8}\right\}+a\left\{\left(\frac{t_{4}^{2}}{2}+\frac{t_{3}^{2}}{2}\right)-r\left(\frac{t_{4}^{3}}{6}+\frac{t_{3}^{3}}{3}\right)+\frac{b}{2}\left(\frac{2 t_{4}^{3}}{3}+\frac{t_{3}^{3}}{3}\right)-\frac{b r}{2}\left(\frac{t_{4}^{4}}{2}+\frac{t_{3}^{4}}{4}\right)\right\}\right] \\
\left.+W\left\{\left(t_{3}-t_{1}\right)-\frac{\alpha t_{3}^{\beta+1}}{\beta+1}-\frac{\alpha t_{1}^{\beta+1}}{\beta+1}\right)\right\}
\end{array}\right.
\end{aligned}
$$

Present worth of Deteriorated items

$$
D=C_{3}\left\{\int_{0}^{t_{1}} \alpha \beta t^{\beta-1} I_{1}(t) e^{-r t} d t+\int_{t_{1}}^{t_{2}} \alpha \beta t^{\beta-1} I_{2}(t) e^{-r t} d t+\int_{t_{2}}^{t_{3}} \alpha \beta t^{\beta-1} I_{3}(t) e^{-r t} d t+\int_{t_{1}}^{t_{3}} \alpha \beta t^{\beta-1} I_{4}(t) e^{-r t} d t \int_{t_{3}}^{t_{4}} \alpha \beta t^{\beta-1} I_{5}(t) e^{-r t} d t\right\}
$$




$$
=\alpha \beta C_{3}\left[\begin{array}{l}
(k-1) a\left\{\frac{t_{1}^{\beta+1}}{(\beta+1)}+\frac{t_{2}^{\beta+1}}{(\beta+1)}-\frac{r t_{1}^{\beta+2}}{(\beta+2)}-\frac{r t_{2}^{\beta+2}}{(\beta+2)}+\frac{b t_{1}^{\beta+2}}{2(\beta+2)}+\frac{b t_{2}^{\beta+2}}{2(\beta+2)}-\frac{b r t_{1}^{\beta+3}}{2(\beta+3)}-\frac{b r t_{2}^{\beta+3}}{2(\beta+3)}\right\} \\
\left.\begin{array}{l}
\frac{t_{3}^{\beta+1}}{\beta(\beta+1)}+\frac{t_{4}^{\beta+1}}{\beta(\beta+1)}+\frac{t_{3}^{\beta+1}}{(\beta+1)}+\frac{t_{2}^{\beta+1}}{(\beta+1)}-\frac{r t_{4}^{\beta+2}}{(\beta+1)(\beta+2)} \frac{r t_{3}^{\beta+2}}{(\beta+2)}-\frac{r t_{3}^{\beta+2}}{(\beta+1)(\beta+2)}-\frac{r t_{2}^{\beta+2}}{(\beta+2)} \\
\frac{b t_{3}^{\beta+2}}{\beta(\beta+2)}+\frac{b t_{4}^{\beta+2}}{\beta(\beta+2)}+\frac{b t_{3}^{\beta+2}}{2(\beta+2)}+\frac{b t_{2}^{\beta+2}}{2(\beta+2)}-\frac{b r t_{1}^{\beta+3}}{2(\beta+3)}-\frac{b r t_{2}^{\beta+3}}{2(\beta+3)}
\end{array}\right\}+ \\
W\left\{\frac{1}{\beta}\left(t_{3}^{\beta}-t_{1}^{\beta}\right)-\frac{r}{\beta+1}\left(t_{3}^{\beta+1}-t_{1}^{\beta+1}\right)\right\}
\end{array}\right\}
$$

Salvage value for Deteriorated Items

$$
\begin{aligned}
& S V=\pi\left\{\int_{0}^{t_{1}} \alpha \beta t^{\beta-1} I_{1}(t) e^{-r t} d t+\int_{t_{1}}^{t_{2}} \alpha \beta t^{\beta-1} I_{2}(t) e^{-r t} d t+\int_{t_{2}}^{t_{3}} \alpha \beta t^{\beta-1} I_{3}(t) e^{-r t} d t+\int_{t_{1}}^{t_{3}} \alpha \beta t^{\beta-1} I_{4}(t) e^{-r t} d t \int_{t_{3}}^{t_{4}} \alpha \beta t^{\beta-1} I_{5}(t) e^{-r t}\right. \\
& =\alpha \beta \pi\left[\begin{array}{l}
(k-1) a\left\{\frac{t_{1}^{\beta+1}}{(\beta+1)}+\frac{t_{2}^{\beta+1}}{(\beta+1)}-\frac{r t_{1}^{\beta+2}}{(\beta+2)}-\frac{r t_{2}^{\beta+2}}{(\beta+2)}+\frac{b t_{1}^{\beta+2}}{2(\beta+2)}+\frac{b t_{2}^{\beta+2}}{2(\beta+2)}-\frac{b r t_{1}^{\beta+3}}{2(\beta+3)}-\frac{b r t_{2}^{\beta+3}}{2(\beta+3)}\right\} \\
+a\left\{\begin{array}{l}
\frac{t_{3}^{\beta+1}}{\beta(\beta+1)}+\frac{t_{4}^{\beta+1}}{\beta(\beta+1)}+\frac{t_{3}^{\beta+1}}{(\beta+1)}+\frac{t_{2}^{\beta+1}}{(\beta+1)}-\frac{r t_{4}^{\beta+2}}{(\beta+1)(\beta+2)}-\frac{r t_{3}^{\beta+2}}{(\beta+2)}-\frac{r t_{3}^{\beta+2}}{(\beta+1)(\beta+2)}-\frac{r t_{2}^{\beta+2}}{(\beta+2)} \\
\frac{b t_{3}^{\beta+2}}{\beta(\beta+2)}+\frac{b t_{4}^{\beta+2}}{\beta(\beta+2)}+\frac{b t_{3}^{\beta+2}}{2(\beta+2)}+\frac{b t_{2}^{\beta+2}}{2(\beta+2)}-\frac{b r t_{1}^{\beta+3}}{2(\beta+3)}-\frac{b r t_{2}^{\beta+3}}{2(\beta+3)}
\end{array}\right\}+ \\
W\left\{\frac{1}{\beta}\left(t_{3}^{\beta}-t_{1}^{\beta}\right)-\frac{r}{\beta+1}\left(t_{3}^{\beta+1}-t_{1}^{\beta+1}\right)\right\}
\end{array}\right]
\end{aligned}
$$

Present worth of Shortage cost

$$
\begin{aligned}
& I_{s}=C_{s}\left[-\int_{t_{4}}^{t_{5}} I_{6}(t) e^{-r t} d t-\int_{t_{5}}^{T} I_{7}(t) e^{-r t} d t\right] \\
& =-a C_{s}\left[\left\{-\frac{t_{4}^{2}}{2}-\frac{t_{5}^{2}}{2}+\frac{r t_{5}^{3}}{3}+\frac{r t_{4}^{3}}{6}+\frac{b \delta t_{5}^{4}}{12}+\frac{b \delta t_{4}^{4}}{4}\right\}+(k-1)\left\{-\frac{T^{2}}{2}-\frac{t_{5}^{2}}{2}+\frac{r T^{3}}{6}-\frac{r t_{5}^{3}}{3}-\frac{b T^{3}}{3}-\frac{b t_{5}^{3}}{6}\right\}\right]
\end{aligned}
$$

Present worth of lost sales quantity

$I_{L}=C_{L} \int_{t_{4}}^{t_{5}}\left[1-e^{-\delta t}\right] a e^{b t} e^{-r t} d t=C_{L} a\left[\frac{\delta}{2}\left(t_{5}^{2}-t_{4}^{2}\right)+\frac{(b-r) \delta}{3}\left(t_{5}^{3}-t_{4}^{3}\right)-\frac{b r \delta}{4}\left(t_{5}^{4}-t_{4}^{4}\right)\right]$

The number of defective items $\mathrm{N}$ in a production cycle is

$$
N=\left\{\begin{array}{cc}
0 & X \geq t_{2} \\
\int_{0}^{t_{2}} k a e^{b t} d t & X \leq t_{2}
\end{array}=t_{2}\left\{\begin{array}{cc}
0 & X \geq t_{2} \\
k a\left(t_{2}+\frac{b t_{2}^{2}}{2}\right) & X \leq t_{2}
\end{array}\right.\right.
$$

The expected number of defective items is

$$
E(N)=\int_{0}^{t_{2}} k a\left(t_{2}+\frac{b t_{2}^{2}}{2}\right)\left(1-r t_{2}\right) f(X) d X
$$

where $f(X)=\mu e^{-\mu X}$

$$
E(N)=k a \mu\left[t_{2}^{2}-r t_{2}^{3}-\frac{\mu t_{2}^{2}}{2}+\frac{b t_{2}^{3}}{2}\right]
$$


Present worth of rework cost

$R C=C_{5} E(N)$

Present worth of Total cost

$T C=\frac{1}{T}\left[P C+H_{R W}+H_{O W}+D+I_{s}+I_{L}+R C-S V\right]$

\subsection{Model 2: When model starts with the shortages}

In Fig.2, the inventory level during a production cycle in which both OW and RW are used. Initially, the inventory level is zero. At this time shortages starts developing and at time $t_{1}$ it reaches to maximum shortage level, at this time fresh production starts to clear the backlog by the time $t_{2}$. The production starts at time $t=t_{2}$ and items accumulate from 0 up to $W$ units in $O W$ in $t_{3}$ units of time. After time $t_{3}$ any production quantity exceeding $\mathrm{W}$ will be stored in RW. After this production stopped and the inventory level in RW begins to decrease at $t_{4}$ and will reach 0 units at $t_{5}$ because of demand and deterioration. The inventory level in OW comes to decrease at $t_{3}$ and then falls below $\mathrm{W}$ at $\mathrm{t}_{5} \mathrm{due}$ to deterioration. The remaining stocks in $\mathrm{OW}$ will be fully exhausted at $\mathrm{T}$ owing to demand and deterioration, the inventory becomes zero.

$$
\begin{array}{ll}
I_{1}^{\prime}(t)=-B d & 0 \leq t \leq t_{1} \\
I_{2}^{\prime}(t)=P-d & t_{1} \leq t \leq t_{2} \\
I_{3}^{\prime}(t)+\alpha \beta t^{\beta-1} I_{3}(t)=P-d & t_{2} \leq t \leq t_{3} \\
I_{4}^{\prime}(t)+\alpha \beta t^{\beta-1} I_{4}(t)=P-d & t_{3} \leq t \leq t_{4} \\
I_{5}^{\prime}(t)+\alpha \beta t^{\beta-1} I_{5}(t)=-d & t_{4} \leq t \leq t_{5} \\
I_{6}^{\prime}(t)+\alpha \beta t^{\beta-1} I_{6}(t)=-d & t_{5} \leq t \leq T \\
I_{7}^{\prime}(t)+\alpha \beta t^{\beta-1} I_{7}(t)=0 & t_{3} \leq t \leq t_{5}
\end{array}
$$

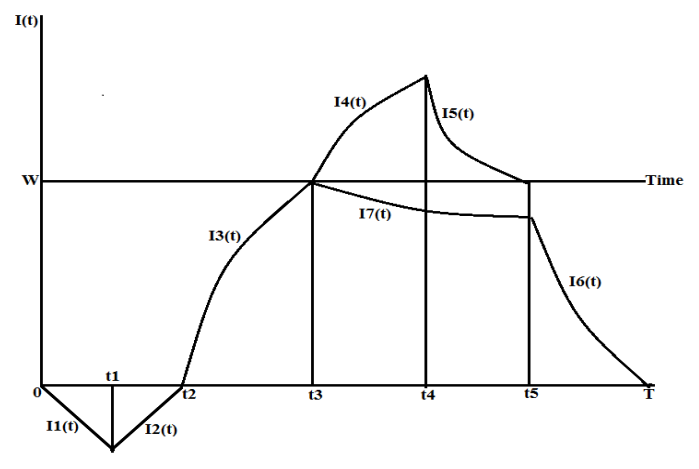

Fig. 2. Two warehouse model which is starts from shortages.

With these boundary conditions

$$
\begin{aligned}
& I_{1}(0)=0, I_{2}\left(t_{2}\right)=0, I_{3}\left(t_{2}\right)=0, I_{4}\left(t_{3}\right)=0, I_{5}\left(t_{5}\right)=0, I_{6}(T)=0, \text { and } I_{7}\left(t_{3}\right)=W \\
& I_{1}(t)=-a\left[t+\frac{(b-\delta) t^{2}}{2}-\frac{b \delta t^{3}}{3}\right] \\
& I_{2}(t)=(k-1) a\left[\left(t-t_{2}\right)+\frac{b}{2}\left(t^{2}-t_{2}^{2}\right)\right] \\
& I_{3}(t)=(k-1) a\left[\left(t-t_{2}\right)+\frac{b}{2}\left(t^{2}-t_{2}^{2}\right)+\frac{a}{\beta+1}\left(t^{\beta+1}-t_{2}^{\beta+1}\right)-\frac{\alpha b}{\beta+2}\left(t^{\beta+2}-t_{2}^{\beta+2}\right)\right]\left(1-\alpha t^{\beta}\right)
\end{aligned}
$$




$$
\begin{aligned}
& I_{4}(t)=(k-1) a\left[\left(t-t_{3}\right)+\frac{b}{2}\left(t^{2}-t_{3}^{2}\right)+\frac{\alpha}{\beta+1}\left(t^{\beta+1}-t_{3}^{\beta+1}\right)+\frac{\alpha b}{\beta+2}\left(t^{\beta+2}-t_{3}^{\beta+2}\right)\right]\left(1-\alpha t^{\beta}\right) \\
& I_{5}(t)=a\left[\left(t_{5}-t\right)+\frac{b}{2}\left(t_{5}^{2}-t^{2}\right)+\frac{\alpha}{\beta+1}\left(t_{5}^{\beta+1}-t^{\beta+1}\right)+\frac{\alpha b}{\beta+2}\left(t_{5}{ }^{\beta+2}-t^{\beta+2}\right)\right]\left(1-\alpha t^{\beta}\right) \\
& I_{6}(t)=a\left[(T-t)+\frac{b}{2}\left(T^{2}-t^{2}\right)+\frac{\alpha}{\beta+1}\left(T^{\beta+1}-t^{\beta+1}\right)+\frac{\alpha b}{\beta+2}\left(T^{\beta+2}-t^{\beta+2}\right)\right]\left(1-\alpha t^{\beta}\right) \\
& I_{7}(t)=W e^{\alpha\left(t_{3}^{\beta}-t^{\beta}\right)}
\end{aligned}
$$

Production cost

$$
P C_{1}=\left(C_{p}+\frac{C_{0}}{n^{\phi}}\right) \int_{t_{2}}^{t_{4}} k a e^{b t} e^{-r t} d t\left(C_{p}+\frac{C_{0}}{n^{\phi}}\right) k\left[a\left(t_{4}-t_{2}\right)+\frac{(b-r)}{2}\left(t_{4}^{2}-t_{2}^{2}\right)\right]
$$

Present worth of Holding cost in $R W$

$$
\begin{aligned}
& H_{1 R W}=C_{R W}\left\{\int_{t_{3}}^{t_{4}} I_{4}(t) e^{-r t} d t+\int_{t_{4}}^{t_{5}} I_{5}(t) e^{-r t} d t\right\} \\
& =a C_{R W}\left[(k-1)\left\{\left(\frac{t_{4}^{2}}{2}+\frac{t_{3}^{2}}{2}\right)-r\left(\frac{t_{4}^{3}}{3}+\frac{t_{3}^{3}}{6}\right)+\frac{b}{2}\left(\frac{t_{4}^{3}}{3}+\frac{2 t_{3}^{3}}{3}\right)\right\}+\left\{\left(\frac{t_{5}^{2}}{2}+\frac{t_{4}^{2}}{2}\right)-r\left(\frac{t_{4}^{3}}{3}+\frac{t_{5}^{3}}{6}\right)+\frac{b}{2}\left(\frac{t_{4}^{3}}{3}+\frac{2 t_{5}^{3}}{3}\right)\right\}\right]
\end{aligned}
$$

Present worth of Holding cost in $\mathrm{OW}$

$$
\begin{aligned}
& H_{1 O W}=C_{O W}\left\{\int_{t_{2}}^{t_{3}} I_{3}(t) d t+\int_{t_{3}}^{t_{5}} I_{7}(t) d t+\int_{t_{5}}^{T} I_{6}(t) d t\right\} \\
& =C_{O W}\left[\begin{array}{l}
\left.(k-1) a\left\{\left(\frac{t_{2}^{2}}{2}+\frac{t_{3}^{2}}{2}\right)-r\left(\frac{t_{2}^{3}}{6}+\frac{t_{3}^{3}}{3}\right)+\frac{b}{2}\left(\frac{2 t_{2}^{3}}{3}+\frac{t_{3}^{3}}{3}\right)-\frac{b r}{2}\left(\frac{t_{2}^{4}}{2}+\frac{t_{3}^{4}}{4}\right)\right\}+a\left\{\begin{array}{l}
\left(\frac{T^{2}}{2}+\frac{t_{5}^{2}}{2}\right)-r\left(\frac{T^{3}}{6}+\frac{t_{5}^{3}}{3}\right) \\
+\frac{b}{2}\left(\frac{2 T^{3}}{3}+\frac{t_{5}^{3}}{3}\right)-\frac{b r}{2}\left(\frac{T^{4}}{2}+\frac{t_{5}^{4}}{4}\right)
\end{array}\right\}\right] \\
+W\left\{\left(t_{5}-t_{3}\right)-\frac{\alpha t_{5}^{\beta+1}}{\beta+1}\right\}
\end{array}\right]
\end{aligned}
$$

Present worth of deteriorated items

$$
\begin{aligned}
& D_{1}=C_{3}\left[\int_{t_{2}}^{t_{3}} \alpha \beta t^{\beta-1} I_{3}(t) d t+\int_{t_{3}}^{t_{4}} \alpha \beta t^{\beta-1} I_{4}(t) d t+\int_{t_{4}}^{t_{5}} \alpha \beta t^{\beta-1} I_{5}(t) d t+\int_{t_{5}}^{T} \alpha \beta t^{\beta-1} I_{6}(t) d t+\int_{t_{3}}^{t_{5}} \alpha \beta t^{\beta-1} I_{7}(t) d t\right] \\
& (k-1) a\left\{\begin{array}{l}
\frac{t_{3}^{\beta+1}}{(\beta+1)}+\frac{t_{2}^{\beta+1}}{\beta(\beta+1)}-\frac{r t_{3}^{\beta+2}}{(\beta+2)}-\frac{r t_{2}^{\beta+2}}{(\beta+1)(\beta+2)}+\frac{b t_{3}^{\beta+2}}{2(\beta+2)}+\frac{b t_{2}^{\beta+2}}{\beta(\beta+2)}-\frac{b r t_{3}^{\beta+3}}{2(\beta+3)}-\frac{b r t_{2}^{\beta+3}}{(\beta+1)(\beta+3)} \\
+\frac{t_{4}^{\beta+1}}{(\beta+1)}+\frac{t_{3}^{\beta+1}}{\beta(\beta+1)}-\frac{r t_{4}^{\beta+2}}{(\beta+2)}-\frac{r t_{3}^{\beta+2}}{(\beta+1)(\beta+2)}+\frac{b t_{4}^{\beta+2}}{2(\beta+2)}+\frac{b t_{3}^{\beta+2}}{\beta(\beta+2)}-\frac{b r t_{4}^{\beta+3}}{2(\beta+3)}-\frac{b r t_{3}^{\beta+3}}{(\beta+1)(\beta+3)}
\end{array}\right.
\end{aligned}
$$

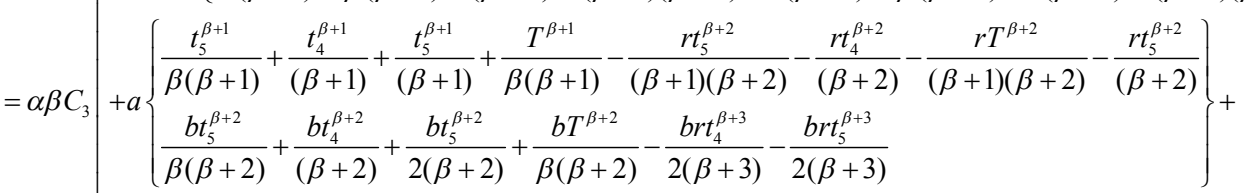

$$
\begin{aligned}
& W\left\{\frac{1}{\beta}\left(t_{5}^{\beta}-t_{3}^{\beta}\right)-\frac{r}{\beta+1}\left(t_{5}^{\beta+1}-t_{3}^{\beta+1}\right)\right\}
\end{aligned}
$$


Salvage value for Deteriorated Items

$$
\begin{aligned}
& S V=\pi\left\{\int_{0}^{t_{1}} \alpha \beta t^{\beta-1} I_{1}(t) e^{-r t} d t+\int_{t_{1}}^{t_{2}} \alpha \beta t^{\beta-1} I_{2}(t) e^{-r t} d t+\int_{t_{2}}^{t_{3}} \alpha \beta t^{\beta-1} I_{3}(t) e^{-r t} d t+\int_{t_{1}}^{t_{3}} \alpha \beta t^{\beta-1} I_{4}(t) e^{-r t} d t \int_{t_{3}}^{t_{4}} \alpha \beta t^{\beta-1} I_{5}(t) e^{-r t} d t\right\} \\
& =\alpha \beta \pi\left[\begin{array}{c}
(k-1) a\left\{\begin{array}{l}
\frac{t_{3}^{\beta+1}}{(\beta+1)}+\frac{t_{2}^{\beta+1}}{\beta(\beta+1)}-\frac{r t_{3}^{\beta+2}}{(\beta+2)}-\frac{r t_{2}^{\beta+2}}{(\beta+1)(\beta+2)}+\frac{b t_{3}^{\beta+2}}{2(\beta+2)}+\frac{b t_{2}^{\beta+2}}{\beta(\beta+2)}-\frac{b r t_{3}^{\beta+3}}{2(\beta+3)}-\frac{b r t_{2}^{\beta+3}}{(\beta+1)(\beta+3)} \\
+\frac{t_{4}^{\beta+1}}{(\beta+1)}+\frac{t_{3}^{\beta+1}}{\beta(\beta+1)}-\frac{r t_{4}^{\beta+2}}{(\beta+2)}-\frac{r t_{3}^{\beta+2}}{(\beta+1)(\beta+2)}+\frac{b t_{4}^{\beta+2}}{2(\beta+2)}+\frac{b t_{3}^{\beta+2}}{\beta(\beta+2)}-\frac{b r t_{4}^{\beta+3}}{2(\beta+3)}-\frac{b r t_{3}^{\beta+3}}{(\beta+1)(\beta+3)}
\end{array}\right\} \\
\left.\begin{array}{l}
\frac{t_{5}^{\beta+1}}{\beta(\beta+1)}+\frac{t_{4}^{\beta+1}}{(\beta+1)}+\frac{t_{5}^{\beta+1}}{(\beta+1)}+\frac{T^{\beta+1}}{\beta(\beta+1)}-\frac{r t_{5}^{\beta+2}}{(\beta+1)(\beta+2)}-\frac{r t_{4}^{\beta+2}}{(\beta+2)}-\frac{r T^{\beta+2}}{(\beta+1)(\beta+2)}-\frac{r t_{5}^{\beta+2}}{(\beta+2)} \\
\frac{b t_{5}^{\beta+2}}{\beta(\beta+2)}+\frac{b t_{4}^{\beta+2}}{(\beta+2)}+\frac{b t_{5}^{\beta+2}}{2(\beta+2)}+\frac{b T^{\beta+2}}{\beta(\beta+2)}-\frac{b r t_{4}^{\beta+3}}{2(\beta+3)}-\frac{b r t_{5}^{\beta+3}}{2(\beta+3)}
\end{array}\right\}+ \\
W\left\{\frac{1}{\beta}\left(t_{5}^{\beta}-t_{3}^{\beta}\right)-\frac{r}{\beta+1}\left(t_{5}^{\beta+1}-t_{3}^{\beta+1}\right)\right\}
\end{array}\right]
\end{aligned}
$$

Present worth of shortage cost

$$
\begin{aligned}
& I_{s}=C_{s}\left[-\int_{0}^{t_{1}} I_{1}(t) e^{-r t} d t-\int_{t_{1}}^{t_{2}} I_{2}(t) e^{-r t} d t\right] \\
& =-a C_{s}\left[\left\{-\frac{t_{1}^{2}}{2}+\frac{r t_{1}^{3}}{3}+\frac{(b-\delta) r t_{1}^{4}}{8}+\frac{b \delta t_{1}^{4}}{12}+\frac{b \delta r t_{1}^{5}}{15}\right\}+(k-1)\left\{-\frac{t_{2}^{2}}{2}-\frac{t_{1}^{2}}{2}+\frac{r t_{2}^{3}}{6}-\frac{r t_{1}^{3}}{3}-\frac{b t_{2}^{3}}{3}-\frac{b t_{1}^{3}}{6}\right\}\right]
\end{aligned}
$$

Present worth of lost sales

$$
I_{L}=C_{L} \int_{0}^{t_{1}}\left[1-e^{-\delta t}\right] a e^{b t} e^{-r t} d t=C_{4} a\left[\frac{\delta}{2} t_{1}^{2}+\frac{(b-r) \delta}{3} t_{1}^{3}-\frac{b r \delta}{4} t_{1}^{4}\right]
$$

Present worth of total cost

$\mathrm{TC}=\frac{1}{T}\left[O C_{1}+H_{1 R W}+H_{1 O W}+D_{1}+I_{s}+I_{L}-S V\right]$

\section{Numerical Example}

For Model 1:

$$
\begin{gathered}
\quad a=250, b=2.2, C_{p}=2.2, k=2, \gamma=0.1, \alpha=0.05, \beta=0.03, W=50, \delta=0.1, C_{R W}=1.9, \\
C_{O W}=1.6, C_{3}=5, \pi=50, C_{S}=5, C_{L}=3, \mu=0.001, C_{5}=0.7, r=0.02 \\
t_{1}^{*}=0.183197, t_{2}^{*}=0.260739, t_{3}^{*}=1.5391, t_{4}^{*}=2.92868, t_{5}^{*}=9.18837, T^{*}=11.2367, T C=13387.1
\end{gathered}
$$

For Model 2:

$$
\begin{aligned}
& a=100, b=1.8, C_{p}=2.2, k=2, \alpha=0.05, \beta=0.03, W=50, \delta=0.1, C_{R W}=1.9, C_{O W}=1.6, \\
& C_{3}=5, \pi=50, C_{s}=5, C_{L}=3, \mu=0.001, C_{5}=0.7, r=0.02
\end{aligned}
$$

Output results

$$
\begin{aligned}
& t_{1}^{*}=1.57369, t_{2}^{*}=2.39014, t_{3}^{*}=2.53839, t_{4}^{*}=6.65712, t_{5}^{*}=8.82671, T^{*}=10.635, \\
& T C=1767.38
\end{aligned}
$$




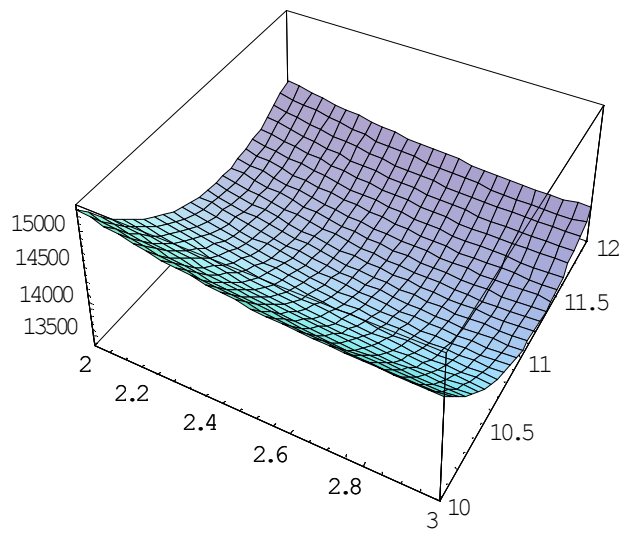

Fig. 3. Convexity of $t_{4} *$ and T w.r.t TC

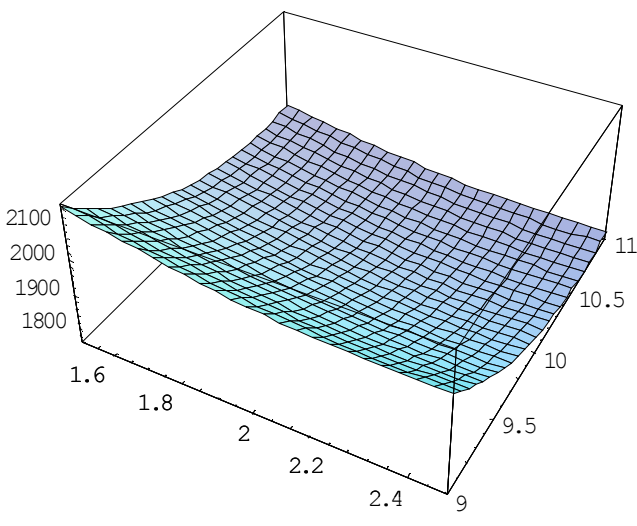

Fig. 4. Convexity of $\mathrm{t}_{2}{ }^{*}$ and $\mathrm{T}^{*}$ w.r.t. $\mathrm{TC}$

\section{Table 1}

Sensitivity analysis of model 1

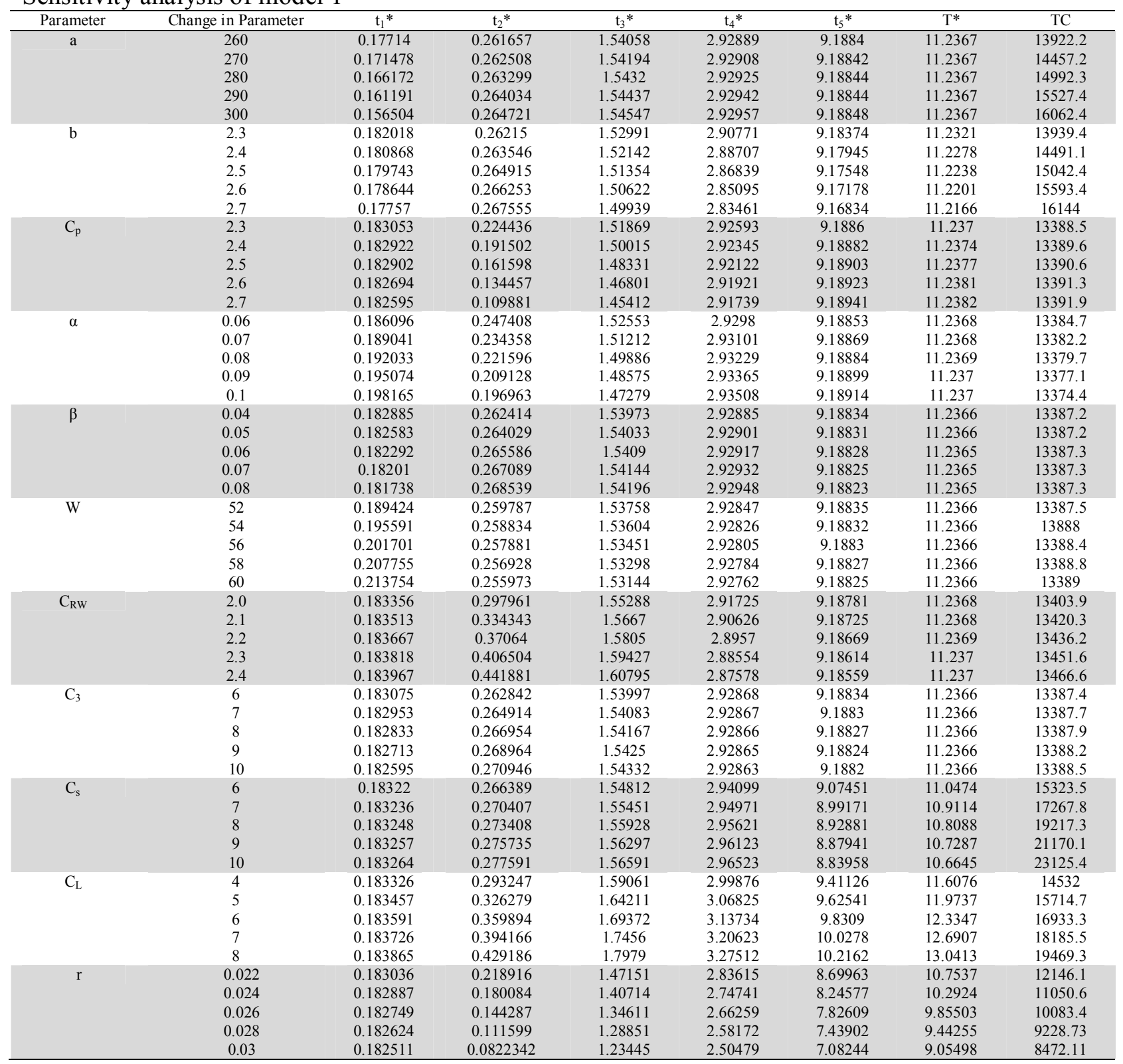


Table 2

Sensitivity analysis of model 2

\begin{tabular}{|c|c|c|c|c|c|c|c|c|}
\hline Parameter & Change in Parameter & $\mathrm{t}_{1} *$ & $\mathrm{t}_{2} *$ & $\mathrm{t}_{3} *$ & $\mathrm{t}_{4} *$ & $t_{5} *$ & $\mathrm{~T}^{*}$ & $\mathrm{TC}$ \\
\hline \multirow[t]{5}{*}{$\mathrm{a}$} & 102 & 1.57369 & 2.39014 & 2.53839 & 6.65712 & 8.82671 & 10.635 & 1801.75 \\
\hline & 104 & 1.5679 & 2.38209 & 2.5252 & 6.6554 & 8.82409 & 10.6306 & 1836.13 \\
\hline & 106 & 1.56515 & 2.37828 & 2.51895 & 6.65458 & 8.82285 & 10.6286 & 1870.5 \\
\hline & 108 & 1.56251 & 2.3746 & 2.51292 & 6.6538 & 8.82166 & 10.6266 & 1904.86 \\
\hline & 110 & 1.55996 & 2.37105 & 2.5071 & 6.65304 & 8.82051 & 10.6247 & 1939.23 \\
\hline \multirow[t]{4}{*}{$\mathrm{C}_{3}$} & 5.2 & 1.57406 & 2.39065 & 2.53923 & 6.65723 & 8.82688 & 10.6352 & 1767.45 \\
\hline & 5.4 & 1.57443 & 2.39117 & 2.54006 & 6.65727 & 8.82705 & 10.6354 & 1767.55 \\
\hline & 5.6 & 1.5748 & 2.39169 & 2.5409 & 6.65773 & 8.82721 & 10.6355 & 1767.65 \\
\hline & 6.0 & 1.57555 & 2.39272 & 2.54257 & 6.65789 & 8.82754 & 10.6359 & 1767.85 \\
\hline \multirow[t]{5}{*}{$\mathrm{C}_{\mathrm{s}}$} & 5.2 & 1.45436 & 2.22143 & 2.37725 & 6.64029 & 8.8097 & 10.6181 & 1776.85 \\
\hline & 5.4 & 1.3453 & 2.0666 & 2.23002 & 6.62487 & 8.79407 & 10.6024 & 1785.09 \\
\hline & 5.6 & 1.24482 & 1.92335 & 2.09444 & 6.61057 & 8.77957 & 10.5876 & 1792.28 \\
\hline & 5.8 & 1.15156 & 1.78983 & 1.96874 & 6.59721 & 8.76958 & 10.5735 & 1798.58 \\
\hline & 6.0 & 1.06439 & 1.6645 & 1.85141 & 6.58458 & 8.75313 & 10.56 & 1804.11 \\
\hline \multirow[t]{4}{*}{$\mathrm{C}_{\mathrm{L}}$} & 4 & 1.50156 & 2.31125 & 2.46294 & 6.64747 & 8.81691 & 10.624 & 1770.47 \\
\hline & 5 & 1.43724 & 2.24092 & 2.39579 & 6.63885 & 8.80813 & 10.6142 & 1773.22 \\
\hline & 7 & 1.32684 & 2.12022 & 2.28086 & 6.62403 & 8.79304 & 10.5974 & 1777.7 \\
\hline & 8 & 1.27892 & 2.06782 & 2.23109 & 6.6176 & 8.78648 & 10.59 & 1779.92 \\
\hline \multirow[t]{5}{*}{$r$} & 0.022 & 1.30904 & 2.09852 & 2.25131 & 6.32867 & 8.28486 & 10.25 & 1627.36 \\
\hline & 0.024 & 1.32511 & 2.13369 & 2.28271 & 5.6715 & 7.79891 & 9.59038 & 1492.31 \\
\hline & 0.026 & 1.34888 & 2.16748 & 2.3105 & 5.29331 & 7.40159 & 9.18875 & 1381.16 \\
\hline & 0.028 & 1.37346 & 2.20234 & 2.33988 & 4.962 & 7.05203 & 8.83589 & 1283.53 \\
\hline & 0.03 & 1.39911 & 2.23866 & 2.37114 & 4.66914 & 6.74171 & 8.52317 & 1196.83 \\
\hline
\end{tabular}

\section{Observations}

1. With increase in demand parameter $a, t_{1}^{*}$ decrease and $a, t_{2}^{*}, t_{3}^{*}, t_{4}^{*}, t_{5}^{*}, T^{*}$ and total cost increases.

2. With increase in demand parameter $b, t_{2}^{*}$ decrease and $t_{1}^{*}, t_{3}^{*}, t_{4}^{*}, t_{5}^{*}, T^{*}$ and total cost increases.

3 . With increase in production cost $C_{p}, t_{1}^{*}, t_{2}^{*}, t_{3}^{*}, t_{4}^{*}, t_{5}^{*}$ decreases and $t_{5}^{*}, T^{*}$ and total cost slightly increases.

4. With increase in warehouse capacity $W, t_{3}^{*}, t_{4}^{*}, t_{5}^{*}$ decreases and $t_{1}^{*}, t_{2}^{*}$ increases and $T^{*}$ and total cost slightly increases.

5. With increase in holding cost of $R W, t_{4}^{*}, t_{5}^{*}$ decreases and $t_{1}^{*}, t_{2}^{*}, t_{3}^{*}, T^{*}$ and total cost increases.

6. With increase in deterioration cost $C_{3}, t_{1}^{*}, t_{4}^{*}, t_{5}^{*}$ decreases and $t_{2}^{*}, t_{3}^{*}, T^{*}$ and total cost increases.

7. With increase in shortage cost of $C_{s}, t_{5}^{*}, T^{*}$ decreases and $t_{1}^{*}, t_{2}^{*}, t_{3}^{*}, t_{4}^{*}$, and total cost increases.

8. With increase in lost sale cost of $C_{L}, t_{1}^{*}, t_{3}^{*}, t_{4}^{*}, t_{5}^{*}, T^{*}$ and total cost increases.

\section{Conclusion}

In this paper, we developed an imperfect quality items with learning and inflation under two storage capacity. We assumed two cases in this paper (i) model ends with shortages (ii) model starts with shortages. Demand is taken as time dependent and dependent on the production. Deterioration is taken as Weibull distribution in both OW and RW. Shortages are allowed and partially backlogged. The effect of learning on production cost is also considered. Learning from one cycle to other cycle, improve the efficiency of the organization. Fig 3 shows the convexity of total cost function for model I. Fig 4 shows the convexity of total cost function for model II. Table 1 and Table 2 show the sensitivity analysis for model 1 and 2, respectively. This paper can be further extended in so many ways: permissible delay, fuzzy environment etc.

\section{References}

Benkherouf, L. (1997). A deterministic order level inventory model for deteriorating items with two storage facilities. International Journal of Production Economics, 48, 167-175.

Bhunia, A.K., \& Maiti, M. (1998). A two-warehouse inventory model for deteriorating items with a linear trend in demand and shortages. Journal of the Operational Research Society, 49, 287-292.

Chan, W.M., Ibrahim, R.N., \& Lochert, P.B. (2003).A new EPQ model: Integrating lower pricing, rework and reject situations. Production Planning and Control, 14(7), 588-595. 
Chung, K. J., \& Hou, K.L. (2003). Optimal productions run time with imperfect production processes and allowable shortages. Computers and Operations Research, 20, 483-490.

Chung, K.-J., \& Huang, Y.F. (2006). Retailer's optimal cycle times in the EOQ model with imperfect quality and a permissible credit period. Quality and Quantity, 40(1), 59-77.

Chung K.J, Her C. C., \& Lin S. D. (2009). A two warehouse inventory model with imperfect quality production processes. Computers \& Industrial Engineering, 56(1), 193-197.

Eroglu, A., \& Ozdemir, G. (2007). An economic order quantity model with defective items and shortages. International Journal of Production Economics, 106(2), 544-549.

Goyal, S.K., \& Giri, B.C. (2003). The production-inventory problem of a product with time varying demand, production and deterioration rates. European Journal of Operational Research, 147, 549-557.

Goyal, S.K., Eduardo, L., \& Barron, C. (2002)."Note on: Economic production quantity model for items with imperfect quality-a practical approach". International Journal of Production Economics 77, 8587.

Goyal, S.K., \& Nebebe, F. (2002). Note on: Economic production quantity model for items with imperfect quality- a practical approach. International Journal of Production Economics, 77, 85-87.

Hartely, R.V. (1976). Operations Research-A Managerial Emphasis. Good Year Publishing Company, California, 315-317.

Huang, C.K. (2004). An optimal policy for a single-vendor single-buyer integrated production-inventory problem with process unreliability consideration. International Journal of Production Economics, 91(1), 91-98.

Hsu, L.-F. (2012). A note on "an economic order quantity (EOQ) for items with imperfect quality and inspection errors". International Journal of Industrial Engineering Computations. 3(4), 695-702.

Jaber, M.Y., Goyal, S.K., \& Imran, M. (2008). Economic production quantity model for items with imperfect quality subject to learning effects. International Journal of Production Economics, 115, 143150.

Jaggi, C.K., \& Verma, P. (2010). Two-warehouse inventory model for deteriorating items with linear trend in demand and shortages under inflationary conditions. International Journal of Procurement Management, 3(1), 54-71.

Kim, C.H., \& Hong, Y. (1999). An optimal production run length in deteriorating production processes. International Journal of Production Economics, 58, 183-189.

Pakkala, T.P.M., \& Achary, K.K. (1992). A deterministic inventory model for deteriorating items with two warehouses and finite replenishment rate. European Journal of Operational Research, 57, 71-76.

Papachristos, S., \& Konstantaras, I. (2006). Economic ordering quantity models for items with imperfect quality. International Journal of Production Economics, 100, 148-154.

Porteus, E.L. (1986). Optimal lot sizing, process quality improvement and setup cost reduction. Operations Research, 18,137-144.

Rosenblatt, M.J., \& Lee, H.L. (1986). Economic production cycles with imperfect production processes. IIE Transactions 18, 48-55.

Sarma, K.V.S. (1987). A deterministic order level inventory model for deteriorating items with two storage facilities. European Journal of Operational Research, 29, 70-73.

Salameh, M.K., \& Jaber, M.Y. (2000). Economic production quantity model for item imperfect quality. International Journal of Production Economics, 64, 59-64.

Singh S.R., Kumar N., \& Kumari R. (2008). Two-Warehouse inventory model for deteriorating items with partial backlogging under the conditions of permissible delay in payments. International Transactions in Mathematical Sciences \& Computer, 1(1), 123-134.

Singh S.R., Kumar N., \& Kumari R. (2009). Two-warehouse Inventory Model for Deteriorating Items with Shortages under Inflation and Time-value of Money. International Journal of Computational and Applied Mathematics, 4 (1), 83-94.

Singh S.R., Jain S., \& Pareek S. (2012). A warehouse imperfect fuzzified production model with shortages and inflation". Advances in Decision Sciences, Volume 2012, Article ID 638060, 16 pages, doi:10.1155/2012/638060.

Wee, H.M., Yu, J., \& Chen, M.C. (2007). Optimal inventory model for items with imperfect quality and shortage backordering. Omega, 35(1), 7-11. 